\title{
Reversine, a selective MPS1 inhibitor, induced autophagic cell death via diminished glucose uptake and ATP production in cholangiocarcinoma cells
}

\author{
Piya Prajumwongs ${ }^{1,2}$, Orawan Waenphimai ${ }^{1,2}$, Kulthida Vaeteewoottacharn ${ }^{1,2}$, Sopit Wongkham ${ }^{1,2}$, Kanlayanee \\ Sawanyawisuth ${ }^{\text {Corresp. 1,2 }}$ \\ ${ }^{1}$ Department of Biochemistry, Faculty of Medicine, Khon Kaen University, Khon Kaen, Thailand \\ 2 Cholangiocarcinoma Research Institute, Faculty of Medicine, Khon Kaen University, Khon Kaen, Thailand \\ Corresponding Author: Kanlayanee Sawanyawisuth \\ Email address: kanlayanee@kkumail.com
}

Reversine is a selective inhibitor of mitotic kinase monopolar spindle 1 (MPS1) and has been reported as an anticancer agent in various cancers. The effects of reversine on bile duct cancer, cholangiocarcinoma (CCA), a lethal cancer in Northeastern Thailand, were investigated. This study reports that reversine inhibited cell proliferation of CCA cell lines in dose- and time-dependent manners but had less inhibitory effect on an immortalized cholangiocyte cell line. Reversine also triggered apoptotic cell death by decreasing antiapoptotic proteins, Bcl-XL and $\mathrm{Mcl}-1$, increasing Bax pro-apoptotic protein and activating caspase-3 activity. Moreover, reversine induced autophagic cell death by increasing LC3-II and Beclin 1 while decreasing p62. Reversine activated autophagy via the AKT signaling pathway. Additionally, this study demonstrated for the first time that reversine could diminish the expression of Hypoxia-Inducible Factor 1- alpha (HIF-1 $\alpha$ ) and glucose transporter 1 (GLUT1), resulting in a reduction of glucose uptake and energy production in CCA cell lines. These findings suggest that reversine could be a good candidate as an alternative or supplementary drug for CCA treatment. 


\section{Reversine, a selective MPS1 inhibitor, induced autophagic}

\section{2 cell death via diminished glucose uptake and ATP}

\section{3 production in cholangiocarcinoma cells}

4 Piya Prajumwongs ${ }^{1,2}$, Orawan Waenphimai ${ }^{1,2}$, Kulthida Vaeteewoottacharn ${ }^{1,2}$, Sopit

5 Wongkham ${ }^{1,2}$, Kanlayanee Sawanyawisuth*1,2

6

$7{ }^{1}$ Department of Biochemistry, Faculty of Medicine, Khon Kaen University, Khon Kaen,

8 Thailand

$9{ }^{2}$ Cholangiocarcinoma Research Institute, Faculty of Medicine, Khon Kaen University, Khon

10 Kaen, Thailand

11

$12 *$ Corresponding Author:

13 Kanlayanee Sawanyawisuth

14 Email address: kanlayanee@kkumail.com

15

16 


\section{Abstract}

18 Reversine is a selective inhibitor of mitotic kinase monopolar spindle 1 (MPS1) and has been 19 reported as an anticancer agent in various cancers. The effects of reversine on bile duct cancer, 20 cholangiocarcinoma (CCA), a lethal cancer in Northeastern Thailand, were investigated. This 21 study reports that reversine inhibited cell proliferation of CCA cell lines in dose- and time-

22 dependent manners but had less inhibitory effect on an immortalized cholangiocyte cell line.

23 Reversine also triggered apoptotic cell death by decreasing anti-apoptotic proteins, Bcl-XL and

24 Mcl-1, increasing Bax pro-apoptotic protein and activating caspase-3 activity. Moreover, 25 reversine induced autophagic cell death by increasing LC3-II and Beclin 1 while decreasing p62.

26 Reversine activated autophagy via the AKT signaling pathway. Additionally, this study

27 demonstrated for the first time that reversine could diminish the expression of Hypoxia-Inducible 28 Factor 1- alpha (HIF-1 $\alpha$ ) and glucose transporter 1 (GLUT1), resulting in a reduction of glucose 29 uptake and energy production in CCA cell lines. These findings suggest that reversine could be a 30 good candidate as an alternative or supplementary drug for CCA treatment. 


\section{Introduction}

33 Cholangiocarcinoma (CCA) is a cancer of bile duct epithelia. Problems of CCA are a high

34 mortality rate related to late diagnoses and cancer metastasis. The highest incidence of CCA has

35 been reported in Northeastern Thailand (Sripa et al. 2007; Sripa \& Pairojkul 2008). The liver

36 fluke, Opisthorchis viverrini, is a major contributing cause of Thai CCA patients (Watanapa \&

37 Watanapa 2002). 5-Fluorouracil (5-FU), the first line chemotherapy drug, is a choice of

38 treatment for unresectable CCA patients, but it is not satisfactory. The outcome is often sub-

39 optimal because CCA patients are commonly intrinsically resistant over time or will become

40 refractory after initial 5-FU treatment, leading to CCA recurrence (Ramirez-Merino et al. 2013).

41 Therefore, the difficulty in these CCA patients is their poor response to the present

42 chemotherapy; studies to identify new therapeutic drugs are therefore needed for therapy.

43 Reversine, a substituted purine derivative, is a selective inhibitor for monopolar spindle 1 (MPS1

44 or TTK), a mitotic checkpoint protein (Hiruma et al. 2016; Hiruma et al. 2017; Santaguida et al.

45 2010). This synthetic compound acts as an adenosine triphosphate (ATP) analogue which

46 inhibits kinase enzyme activity (Hiruma et al. 2016). Monopolar spindle protein 1

47 (MPS1) kinase has been reported to promote progression of cancers and MPS1 overexpression

48 was related with poor survival of cancer patients including breast, liver, lung cancers (Choi et al.

49 2017; King et al. 2018; Tsai et al. 2020; Xu et al. 2016). Laboratory studies revealed that

50 reversine has been shown to inhibit the growth of multiple cancers including colon (Park et al.

51 2019), prostate (Hsieh et al. 2007), oral squamous (Lee et al. 2012a) and thyroid (Lu et al. 2012).

52 The effects of reversine on cancer cells were G2/M cell cycle arrest, autophagy, and apoptosis in

53 those cancers. Reversine also inhibited the phosphatidylinositol 3-kinase/protein kinase B

54 (PI3K/AKT) signaling pathway which plays a role in biological processes including cell growth, 
55 anti-apoptosis and metabolism in cancer cells (Fresno Vara et al. 2004; Thorpe et al. 2015). This

56 inhibitory effect of PI3K/AKT signaling by reversine resulted in the inductions of autophagy and

57 apoptosis (Lee et al. 2012b) (Kumar et al. 2014; Shao et al. 2016). PI3K/AKT regulated GLUT-1

58 and HIF-1 $\alpha$ expressions; modulation of this signaling pathway led to cellular starvation including

59 reduction of glucose uptake and ATP production (Melstrom et al. 2008; Zhang et al. 2016).

60 Upregulation of MPS1 was reported in OV-associated CCA tissues (Jinawath et al. 2006),

61 therefore inhibition of MPS1 activity by reversine may block the G2/M phase of the cell cycle

62 and induce cell death. Reversine may be an alternative choice for CCA treatment. This study

63 aimed to investigate the effect of reversine on cell proliferation in CCA cells and related

64 molecular mechanisms.

65 In the current study, the suppressive effects of reversine on viability of CCA cell lines were

66 determined using the MTT assay, the cell cycle was analyzed by flow cytometry and the related

67 molecular mechanisms of autophagy and apoptosis were investigated using Western blot

68 analysis. The glucose uptake and ATP production were also measured. Results revealed that

69 reversine induced $\mathrm{G} 2 / \mathrm{M}$ arrest, apoptosis and autophagy via glucose and ATP deprivation in

70 CCA cell lines. 


\section{Materials \& Methods}

\section{Cell lines and reagent}

74 CCA cell lines (KKU-100, KKU-213A and KKU-213B) were obtained from the Japanese

75 Collection of Research Bioresources Cell Bank, Osaka, Japan and established as previously 76 described (Sripa et al. 2005; Sripa et al. 2020). An immortalized cholangiocyte cell line,

77 MMNK1 was previously characterized (Maruyama et al. 2004). Cells were cultured in high

78 glucose (25 mM) Dulbecco's Modified Eagle Medium (DMEM) supplemented with 10\% heat

79 inactivated fetal bovine serum (FBS) and a 1\% antibiotic-antimycotic (Gibco, USA). Cells were

80 incubated at $37{ }^{\circ} \mathrm{C}$ in a humidified $5 \% \mathrm{CO}_{2}$ atmosphere. At $70-80 \%$ confluence, cells were

81 detached from the culture flask using $0.25 \% \mathrm{w} / \mathrm{v}$ trypsin/EDTA and processed according to the

82 particular assay. Reversine was purchased from Cayman Chemical (Michigan, USA) and 83 prepared as a $100 \mathrm{mM}$ stock in DMSO.

84

\section{Cell proliferation assay}

86 Cells were seeded in 96 well plates and treated with $0.1 \%$ DMSO as a vehicle control and

87 various concentrations of reversine at $0.1,0.5,1,10$ and $20 \mu \mathrm{M}$, for 24,48 , and $72 \mathrm{~h}$. MTT

88 reagent was added into each well to a final concentration of $0.5 \mathrm{mg} / \mathrm{ml}$ and incubated for $4 \mathrm{~h}$.

89 DMSO was added to dissolve the insoluble formazan complex and the absorbance at $540 \mathrm{~nm}$ was

90 measured. The data were analyzed at a half maximal inhibitory concentration $\left(\mathrm{IC}_{50}\right)$ by the dose-

91 response inhibition mode of the GraphPad Prism ${ }^{\circledR} 7.0$ software (GraphPad software, Inc., CA, 92 USA). 


\section{Cell cycle analysis}

95 KKU-213A and KKU-213B cell lines $\left(2 \times 10^{5}\right.$ cells $)$ were seeded into a 6 -well plate and treated

96 with reversine $(0,1,2$ and $4 \mu \mathrm{M})$ for $24 \mathrm{~h}$. Cells were collected, fixed with cold $70 \%$ ethanol

97 overnight at $4^{\circ} \mathrm{C}$ and treated with $0.1 \mathrm{mg} / \mathrm{ml}$ of RNase $\mathrm{A}$ at $37^{\circ} \mathrm{C}$ for $1 \mathrm{~h}$. Cells were stained with

$9810 \mu \mathrm{g} / \mathrm{ml}$ propidium iodide (PI) at $4^{\circ} \mathrm{C}$ for $30 \mathrm{~min}$ in the dark. Cell cycle analysis was determined

99 using a flow cytometer (BD LSR II $\left.{ }^{\mathrm{TM}}\right)$. Flow $\mathrm{Jo}^{\mathrm{TM}}$ software was used to generate the acquisition 100 and analysis plots of 20,000 cells.

101

102

\section{Western blot analysis}

103 CCA cells were lysed in buffer (50 mM Tris-HCl pH 7.5, $50 \mathrm{mM} \mathrm{NaCl,} \mathrm{1 \% NP-40,} \mathrm{1 \%} \mathrm{sodium}$ 104 deoxycholate) containing phosphatase and protease inhibitors. Protein lysates were applied on a 105 12\% SDS-PAGE for Western blot analysis. Proteins were detected by antibodies including PI3K, 106 AKT, phospho-AKT, GLUT1, LC3-II, Mcl-1, and cleaved caspase-3 (Cell Signaling

107 Technology, USA), HIF-1 $\alpha$ (BD biosciences, USA), Bcl-XL, and Bax (Santa Cruz

108 Biotechnology, USA), Beclin 1 and p62 (Abcam, USA). GAPDH, (EMD Millipore, Germany)

109 was used as an internal control. The immunoreactivity signals were captured and analyzed by 110 ImageQuant $^{\mathrm{TM}}$ TL analysis software (GE Healthcare, UK).

\section{Glucose and ATP measurement}

KKU-213A and KKU-213B cell lines $\left(5 \times 10^{5}\right.$ cells $)$ were seeded onto 6-well plates and

114 treated with a sublethal dose $\left(\mathrm{IC}_{25}\right)$ at $4 \mu \mathrm{M}$, of reversine for 12 and $24 \mathrm{~h}$. Glucose levels in the 115 collected media were determined using a Glucose Colorimetric/ Fluorometric Assay kit (K606, 
116 BioVision, USA) following the manufacturer's protocol. Absorbance was measured at $570 \mathrm{~nm}$ and

117 the glucose standard provided with the kit was used to calculate glucose levels. The glucose uptake

118 was calculated by comparing the difference in leftover glucose concentrations between the vehicle

119 and the reversine treatment. The amounts of ATP in cell lysates were measured and calculated

120 from the standard curves using the ATP Colorimetric/Fluorometric Assay Kit according to the

121 manufacturer's protocol (K354, BioVision, USA). The glucose uptake and ATP production of 122 vehicle control were set as $100 \%$.

123

124 Statistical analysis

125 Data were presented as mean \pm SD. Statistical comparisons between groups were tested using the 126 Student's $t$-test. The data were analyzed by GraphPad Prism ${ }^{\circledR} 7.0$ software (GraphPad software, 127 Inc., CA, USA) and SPSS 17.0 software (IL, USA). A $P$-value less than 0.05 was considered as 128 statistically significant.

129

130 Results

131 Reversine inhibited cell proliferation in CCA cell lines

132 Three CCA cell lines, KKU-100, KKU-213A and KKU-213B, and an immortalized

133 cholangiocyte cell line, MMNK1, were treated with various concentrations of reversine $(0,0.1$,

$1340.5,1$, and $10 \mu \mathrm{M}$ ) for 24,48 and $72 \mathrm{~h}$. Cell viability was significantly decreased in dose- and 135 time-dependent manners in all cell lines $\left({ }^{*} P<0.05\right)$ (Fig. 1A-C). The half-maximal inhibitory 136 concentrations $\left(\mathrm{IC}_{50}\right)$ of reversine at different time points for the $3 \mathrm{CCA}$ cell lines were in the $137 \quad 0.62-10 \mu \mathrm{M}$ range which were significantly lower than those of the MMNK1 cell line (7.05-20 $138 \mu \mathrm{M}$ range) as shown in Table 1. These results showed that reversine potentially reduced the cell 
139 viability of the CCA cell lines whereas it had less effect on the MMNK1 cell line. In addition,

140 comparison between the cytotoxicity of reversine and 5-FU in CCA cell lines showed that the

$141 \mathrm{IC}_{50}$ of reversine was lower than 5-FU at least 6-12 times (Supplementary Fig. S1 and Table S1).

142 This indicated that reversine was an effective drug and could be an alternative option for CCA

143 treatment.

144

145 Reversine induced cell cycle arrest at the G2/M phase and apoptosis

146 The effects of reversine on the cell cycle were examined in KKU-213A and KKU-213B cell

147 lines that were treated with increasing concentrations of reversine for $24 \mathrm{~h}$ and analyzed by flow

148 cytometry (Fig. 2A). The results demonstrated that reversine significantly decreased the G1

149 phase and induced G2/M arrest in a dose-dependent manner. Reversine tended to increase the 150 sub G1 population (apoptotic cells) in both cell lines as shown in Fig. 2B-C. To confirm these

151 phenomena, a Western blot assay was performed to check the protein expression levels related to $152 \mathrm{G} 2 / \mathrm{M}$ arrest (cyclin B1, p21) and apoptosis (Bcl-XL and Mcl-1, Bax and cleaved caspase-3).

$153 \mathrm{KKU}-213 \mathrm{~A}$ and KKU-213B CCA cell lines were treated with $4 \mu \mathrm{M}$ of reversine at different time 154 points $(12,24,48$ and $72 \mathrm{~h})$. The Western blot results showed a substantial decline of cyclin B1 155 while a significant rise in p21 in reversine treatment occurred. Furthermore, anti-apoptotic 156 proteins including Bcl-XL and Mcl-1 were significantly decreased $(* P<0.05)$, whereas Bax pro157 apoptotic protein and cleaved caspase-3 were increased noticeably in reversine treatment for 48 158 and $72 \mathrm{~h}$ in both CCA cell lines (Fig. 2D-F).

159

160 
161 Reversine induced autophagy and reduced glucose uptake and ATP production via down162 regulation of HIF-1 $\alpha$ and GLUT1

163 The accumulation of cytoplasmic vacuoles that were similar to the morphological characteristics 164 of autophagy were observed in reversine-treated KKU-213A and KKU-213B cell lines at 12 and $16524 \mathrm{~h}$ (Fig. 3A). The expression of LC3-II, Beclin 1 and p62 proteins which are autophagy related 166 markers, were examined in KKU-213A and KKU-213B cell lines treated with $4 \mu \mathrm{M}$ of reversine 167 for 12-72 h (Fig. 3B). A significant increase in LC3-II and Beclin 1 coupled with a decrease in 168 p62 were shown in reversine treatment compared to vehicle controls $(P<0.05)$ (Fig. 3C-D). 169 These results suggested that reversine induced autophagy activation and autophagic flux occurred. To determine whether autophagic cell death induced by reversine treatment altered

171 glucose metabolism, the glucose uptake and ATP production in reversine-treated CCA cells were 172 examined. KKU213A and KKU213B CCA cells were treated with $4 \mu \mathrm{M}$ reversine at 12 and 24 173 h. The cultured media were collected to measure the glucose uptake while the cell lysates were 174 used to measure the ATP production. Reversine treatment significantly reduced glucose uptake 175 at $24 \mathrm{~h}$ and reduced ATP production at 12 and $24 \mathrm{~h}$ as shown in Fig. 3E-F. The protein 176 expression of Hypoxia-inducible factor 1-alpha (HIF-1 $\alpha$ ) and glucose transporter 1 (GLUT1) by 177 Western blot assay were further analyzed. The results showed markedly decreased protein 178 expression of HIF-1 $\alpha$ and GLUT1 in reversine treated cells compared with vehicle control (Fig. 179 3B-D). Collectively, these results indicated that reversine treatment induced autophagic cell 180 death and interfered with glucose uptake and ATP production via reduction of HIF-1 $\alpha$ and 181 GLUT1 protein expression levels.

\section{Reversine induced autophagy via the AKT signaling pathway}


184 The PI3K/AKT signaling pathway has been reported to regulate the autophagy. Therefore, the 185 activation of PI3K/ AKT after reversine treatment was determined by Western blot analysis. Fig. 186 3B-D demonstrated a significant decreased phosphorylation of the p1 $10 \alpha$ catalytic subunit of 187 PI3K (p110 $\alpha)$ and the phosphorylation of serine 473 of AKT (s473) in reversine-treated CCA 188 cell lines.

189 To further confirm that reversine induced autophagy via the AKT signaling pathway, SC-79, an 190 AKT activator was used to activate the phosphorylation of AKT (s473). A combination of 191 reversine and SC-79 recovered the cells from reversine-mediated autophagy (Fig. 4A-B).

192 Western blot results showed a tendency to increase pAKT, HIF-1 $\alpha$, GLUT1 and p62 expression 193 with the concurrence of decreased Beclin 1 and LC3-II in reversine combined with SC-79 194 treatment (Fig. 4C). Among these, LC3-II was significantly decreased in the combination when 195 compared to reversine treatment alone (Fig. 4D-E, ${ }^{\#} P<0.05$ ).

196

\section{Discussion}

198 In this study, it was revealed for the first time that reversine could induce G2/M arrest, apoptosis 199 and autophagic cell death in CCA cell lines. Reversine exhibited an anti-proliferative effect on 200 all CCA cell lines tested in dose and time dependent manners. The $\mathrm{IC}_{50}$ of reversine at $72 \mathrm{~h}$ in

201 Table 1 suggested that reversine had more effect (4 to 11-fold) on CCA cell lines than on the 202 immortalized cholangiocyte. Noteworthy, KKU-100, a 5-FU resistant cell line was more 203 sensitive to reversine than KKU-213A and KKU-213B (Supplementary Table S1). This 204 observation may be due to the alteration of some proteins after reversine treatment that may be 205 involved in the cell viability of each cell line. Further investigation is required to determine the 206 mechanisms which cause some chemo-resistant cell lines to be more sensitive than others to 
207 reversine. The above observation implies that reversine may be a potent anticancer drug for the 208 chemotherapy resistant CCA cells. The effect and mechanism of reversine or in combination 209 with a chemotherapy drug on chemotherapy resistant CCA cells should be interesting to explore. 210 Furthermore, CCA cell lines were more sensitive to reversine than breast cancer cell lines $\left(\mathrm{IC}_{50}\right.$ $211 \sim 5 \mu \mathrm{M})$ (Kuo et al. 2014) and cervical carcinoma cell lines ( $\left.\mathrm{IC}_{50} \sim 7.5 \mu \mathrm{M}\right)$ (Qin et al. 2013). This 212 comparison hints that a low dose of reversine effectively inhibits the proliferation of CCA cells 213 and may be a potential drug for CCA treatment or to augment current treatment. The antitumor 214 activity of reversine in an animal model should be worth further investigation.

215 The growth inhibitory effect of reversine on G2/M cell cycle arrest in CCA cell lines 216 corresponded with the observations in several cancer cells after reversine treatment (Alves et al. 217 2016; D'Alise et al. 2008; Kuo et al. 2014; Lee et al. 2012a; Lu et al. 2012). Western blot 218 analysis supported the $\mathrm{G} 2 / \mathrm{M}$ arrest in reversine-treated cells via the alteration of cyclin B1 and 219 the cyclin-dependent kinase inhibitor (p21) which are the G2/M checkpoint markers. Similarly, 220 decreased expression of cyclin B1 and increased expression of p21 during G2/M arrest after 221 reversine treatment were also reported in prostate cancer (Hsieh et al. 2007) and renal cancer 222 (Cheng et al. 2018).

223 The present results showed reversine could induce apoptosis via down-regulation of anti224 apoptotic proteins (Bcl-XL and Mcl-1), up-regulation of pro-apoptotic Bax protein and activation 225 of caspase-3 in both CCA cell lines (Fig. 2D-F). These results were consistent with the 226 observations in oral squamous cell cancer (Lee et al. 2012a), cervical carcinoma (Qin et al. 2013) 227 and non-small cell lung cancer ( $\mathrm{Lu}$ et al. 2016). The PI3K/AKT signaling pathway has been 228 reported to regulate the apoptosis and autophagy (Baxt \& Xavier 2015; Duronio 2008; Jee et al. 229 2002; Qian et al. 2009; Tsuruta et al. 2002; Wu et al. 2009). Therefore, the phosphorylation of 
230 PI3K (p110 $\alpha)$ and AKT (s473) in reversine treated cells were examined. It was found that the

$231 \mathrm{PI} 3 \mathrm{~K} / \mathrm{AKT}$ signaling pathways were inactivated upon reversine treatment. This finding was

232 similar to the studies in oral squamous cell (Lee et al. 2012a) and thyroid cancers (Lu et al.

233 2012). Furthermore, reversine treatment led to the formation of cytoplasmic vacuoles and

234 increased Beclin 1 and LC3-II, while it decreased p62 which indicated autophagy flux in both

235 CCA cell lines. These findings were also found in the thyroid and non-small-cell-lung cancers

236 (Lu et al. 2012; Lu et al. 2016).

237 Many studies have shown that PI3K/AKT signaling controlled glucose metabolism and energy

238 production by regulating the expression of HIF-1 $\alpha$ and GLUT1 (Hao 2015; Katagiri et al. 1996).

239 Whether reversine affected glucose uptake and ATP production in reversine-treated CCA cells

240 was explored in the current study. It is reported for the first time that reversine treatment down-

241 regulated HIF-1 $\alpha$ and GLUT1 protein expression and consequently lowered glucose uptake and

242 ATP production (Fig. 3B-F). Consistently, these data were accompanied with the inactivation of

$243 \mathrm{PI} 3 \mathrm{~K} / \mathrm{AKT}$ signaling in reversine treatment (Fig. 4C-E). Altogether, reversine disturbed the

244 function of the MPS1 mitotic checkpoint protein and also interrupted PI3K/AKT signaling which

245 is a major signal transduction pathway that involves cancer cell survival.

246 The overall anticancer effects and proposed molecular mechanisms of reversine on CCA cells

247 are summarized in Fig. 5. Reversine, which is a selective inhibitor of MPS1, caused G2/M arrest

248 with alteration of cyclin B1 and p21. It also induced apoptosis and autophagy. Various reports

249 have shown that reversine can inhibit the in vitro kinase activity of aurora kinases, Janus Kinase

2502 (JAK2), Rous Sarcoma (SRC) (McMillin et al. 2010) and ribosomal protein S6 kinase beta-1

251 (p70 ${ }^{\mathrm{s} 6 \mathrm{k}}$ ) (Kim et al. 2007). Reversine may hamper the activity of JAK2 or SRC, consequently

252 inhibiting the phosphorylation of PI3K/AKT signaling and resulting in the down-regulation of 
253 HIF-1 $\alpha$ and GLUT1 expression. These phenomena diminish glucose uptake and ATP production

254 and induce starvation-related autophagy. The current study showed that reversine-mediated

255 autophagy inactivated AKT signaling. Moreover, down-regulation of HIF-1 $\alpha$ by reversine

256 suppresses the expression of transcription factors such as NF-kB, SP1 and CREB. This results in

257 apoptosis induction through a decrease of Bcl-XL and Mcl-1 expressions which subsequently

258 inhibit Bax and activated the cleavage of caspase 3.

259

260 Conclusions

261 Reversine markedly inhibited the cell proliferation of CCA cells by multiple mechanisms

262 including G2/M cell cycle arrest, apoptosis and starvation-related autophagy. These new findings

263 suggest that reversine could be a good candidate drug for CCA treatment. The effects of

264 reversine itself or reversine in combination with current chemotherapy drugs are worthy of

265 further preclinical and clinical investigations.

266

267

268

269

270

271 Publication Clinic KKU, Thailand.

\section{Acknowledgements}

Cholangiocarcinoma Research Institute, Faculty of Medicine, Khon Kaen University, Khon Kaen, Thailand. We would like to acknowledge Prof. James A. Will for editing the MS via

272

273 ADDITIONAL INFORMATION AND DECLARATIONS

\section{Funding}

275 This study was co-supported by the research grant from Khon Kaen University (6200020002),

276 and the invitation research grant from the Faculty of Medicine, Khon Kaen University 
277 (IN62110). Piya Prajumwongs was supported by a scholarship from the Postgraduate

278 Scholarship of Faculty of Medicine, Khon Kaen University.

279

280 Competing Interests

281 The authors declare there are no competing interests.

\section{Author Contributions}

283

284

285

286

287

288

289

290

291

292

293

294

295

296

297

298

299

300

301

302

- Piya Prajumwongs performed the experiments, analyzed the data, wrote the paper, prepared figures and/or tables and original draft preparation,

- Orawan Waenphimai performed the experiments, analyzed the data and prepared figures and/or tables

- Kulthida Vaeteewoottacharn analyzed the data and reviewed drafts of the manuscript,

- Sopit Wongkham analyzed the data and reviewed drafts of the manuscript

- Kanlayanee Sawanyawisuth conceived and designed the experiments, performed the experiments, analyzed the data, wrote the manuscript, reviewed drafts of the manuscript, wrote and edited the manuscript and funding acquisition,

- All authors have read and agreed to the published version of the manuscript.

\section{References}

Alves APNR, Machado-Neto JA, Scheucher PS, Paiva HH, Simões BP, Rego EM, and Traina F. 2016. Reversine triggers mitotic catastrophe and apoptosis in K562 cells. Leukemia research 48:26-31.

Baxt LA, and Xavier RJ. 2015. Role of autophagy in the maintenance of intestinal homeostasis. Gastroenterology 149:553-562.

Cheng L, Wang H, Guo K, Wang Z, Zhang Z, Shen C, Chen L, and Lin J. 2018. Reversine, a substituted purine, exerts an inhibitive effect on human renal carcinoma cells via induction of cell apoptosis and polyploidy. Onco Targets Ther 11:1025-1035. 10.2147/OTT.S158198 
303

304

305

306

307

308

309

310

311

312

313

314

315

316

317

318

319

320

321

322

323

324

325

326

327

328

329

330

331

332

333

334

335

336

337

338

339

340

341

342

343

344

345

346

Choi M, Min YH, Pyo J, Lee CW, Jang CY, and Kim JE. 2017. TC Mps1 12, a novel Mps1 inhibitor, suppresses the growth of hepatocellular carcinoma cells via the accumulation of chromosomal instability. Br J Pharmacol 174:1810-1825. 10.1111/bph.13782

D'Alise AM, Amabile G, Iovino M, Di Giorgio FP, Bartiromo M, Sessa F, Villa F, Musacchio A, and Cortese R. 2008. Reversine, a novel Aurora kinases inhibitor, inhibits colony formation of human acute myeloid leukemia cells. Molecular cancer therapeutics 7:1140-1149.

Duronio V. 2008. The life of a cell: apoptosis regulation by the PI3K/PKB pathway. Biochemical Journal 415:333-344.

Fresno Vara JA, Casado E, de Castro J, Cejas P, Belda-Iniesta C, and Gonzalez-Baron M. 2004. PI3K/Akt signalling pathway and cancer. Cancer Treat Rev 30:193-204. 10.1016/j.ctrv.2003.07.007

Hao J. 2015. HIF-1 is a critical target of pancreatic cancer. Oncoimmunology 4:e1026535.

Hiruma Y, Koch A, Dharadhar S, Joosten RP, and Perrakis A. 2016. Structural basis of reversine selectivity in inhibiting Mps1 more potently than aurora B kinase. Proteins 84:17611766. 10.1002/prot.25174

Hiruma Y, Koch A, Hazraty N, Tsakou F, Medema RH, Joosten RP, and Perrakis A. 2017. Understanding inhibitor resistance in Mps1 kinase through novel biophysical assays and structures. J Biol Chem 292:14496-14504. 10.1074/jbc.M117.783555

Hsieh TC, Traganos F, Darzynkiewicz Z, and Wu JM. 2007. The 2,6-disubstituted purine reversine induces growth arrest and polyploidy in human cancer cells. Int J Oncol 31:1293-1300.

Jee S, Chiu H-C, Tsai T-F, Tsai W, Liao Y, Chu C, and Kuo M. 2002. The phosphotidyl inositol 3-kinase/Akt signal pathway is involved in interleukin-6-mediated Mcl-1 upregulation and anti-apoptosis activity in basal cell carcinoma cells. Journal of investigative dermatology 119:1121-1127.

Jinawath N, Chamgramol Y, Furukawa Y, Obama K, Tsunoda T, Sripa B, Pairojkul C, and Nakamura Y. 2006. Comparison of gene expression profiles between Opisthorchis viverrini and non-Opisthorchis viverrini associated human intrahepatic cholangiocarcinoma. Hepatology 44:1025-1038. 10.1002/hep.21330

Katagiri H, Asano T, Ishihara H, Inukai K, Shibasaki Y, Kikuchi M, Yazaki Y, and Oka Y. 1996. Overexpression of catalytic subunit $\mathrm{p} 110 \alpha$ of phosphatidylinositol 3-kinase increases glucose transport activity with translocation of glucose transporters in 3T3-L1 adipocytes. Journal of Biological Chemistry 271:16987-16990.

Kim YK, Choi HY, Kim NH, Lee W, Seo DW, Kang DW, Lee HY, Han JW, Park SW, and Kim SN. 2007. Reversine stimulates adipocyte differentiation and downregulates Akt and p70(s6k) signaling pathways in 3T3-L1 cells. Biochem Biophys Res Commun 358:553558. 10.1016/j.bbrc.2007.04.165

King JL, Zhang B, Li Y, Li KP, Ni JJ, Saavedra HI, and Dong JT. 2018. TTK promotes mesenchymal signaling via multiple mechanisms in triple negative breast cancer. Oncogenesis 7:69. 10.1038/s41389-018-0077-z

Kumar D, Shankar S, and Srivastava RK. 2014. Rottlerin induces autophagy and apoptosis in prostate cancer stem cells via PI3K/Akt/mTOR signaling pathway. Cancer Lett 343:179189. 10.1016/j.canlet.2013.10.003

Peer] reviewing PDF | (2020:05:49288:2:0:NEW 30 Nov 2020) 
347

348

349

350

351

352

353

354

355

356

357

358

359

360

361

362

363

364

365

366

367

368

369

370

371

372

373

374

375

376

377

378

379

380

381

382

383

384

385

386

387

388

389

390

391

Kuo C-H, Lu Y-C, Tseng Y-S, Shi C-S, Chen S-H, Chen P-T, Wu F-L, Chang Y-P, and Lee YR. 2014. Reversine induces cell cycle arrest, polyploidy, and apoptosis in human breast cancer cells. Breast Cancer 21:358-369.

Lee Y-R, Wu W-C, Ji W-T, Chen JY-F, Cheng Y-P, Chiang M-K, and Chen H-R. 2012a. Reversine suppresses oral squamous cell carcinoma via cell cycle arrest and concomitantly apoptosis and autophagy. Journal of biomedical science 19:9.

Lee YR, Wu WC, Ji WT, Chen JY, Cheng YP, Chiang MK, and Chen HR. 2012b. Reversine suppresses oral squamous cell carcinoma via cell cycle arrest and concomitantly apoptosis and autophagy. J Biomed Sci 19:9. 10.1186/1423-0127-19-9

Lu C-H, Liu Y-W, Hua S-C, Yu H-I, Chang Y-P, and Lee Y-R. 2012. Autophagy induction of reversine on human follicular thyroid cancer cells. Biomedicine \& Pharmacotherapy 66:642-647.

Lu Y-C, Lee Y-R, Liao J-D, Lin C-Y, Chen Y-Y, Chen P-T, and Tseng Y-S. 2016. Reversine induced multinucleated cells, cell apoptosis and autophagy in human non-small cell lung cancer cells. PloS one 11:e0158587.

Maruyama M, Kobayashi N, Westerman KA, Sakaguchi M, Allain JE, Totsugawa T, Okitsu T, Fukazawa T, Weber A, and Stolz DB. 2004. Establishment of a highly differentiated immortalized human cholangiocyte cell line with SV40T and hTERT. Transplantation 77:446-451.

McMillin DW, Delmore J, Weisberg E, Negri JM, Geer DC, Klippel S, Mitsiades N, Schlossman RL, Munshi NC, Kung AL, Griffin JD, Richardson PG, Anderson KC, and Mitsiades CS. 2010. Tumor cell-specific bioluminescence platform to identify stroma-induced changes to anticancer drug activity. Nat Med 16:483-489. 10.1038/nm.2112

Melstrom LG, Salabat MR, Ding XZ, Milam BM, Strouch M, Pelling JC, and Bentrem DJ. 2008. Apigenin inhibits the GLUT-1 glucose transporter and the phosphoinositide 3-kinase/Akt pathway in human pancreatic cancer cells. Pancreas 37:426-431. 10.1097/MPA.0b013e3181735ccb

Park YL, Ha SY, Park SY, Choi JH, Jung MW, Myung DS, Kim HS, and Joo YE. 2019. Reversine induces cell cycle arrest and apoptosis via upregulation of the Fas and DR5 signaling pathways in human colorectal cancer cells. Int J Oncol 54:1875-1883. 10.3892/ijo.2019.4746

Qian J, Zou Y, Rahman JS, Lu B, and Massion PP. 2009. Synergy between phosphatidylinositol 3-kinase/Akt pathway and Bcl-xL in the control of apoptosis in adenocarcinoma cells of the lung. Molecular cancer therapeutics 8:101-109.

Qin H-x, Yang J, Cui H-k, Li S-p, Zhang W, Ding X-1, and Xia Y-h. 2013. Synergistic antitumor activity of reversine combined with aspirin in cervical carcinoma in vitro and in vivo. Cytotechnology 65:643-653.

Ramirez-Merino N, Aix SP, and Cortes-Funes H. 2013. Chemotherapy for cholangiocarcinoma: An update. World J Gastrointest Oncol 5:171-176. 10.4251/wjgo.v5.i7.171

Santaguida S, Tighe A, D'Alise AM, Taylor SS, and Musacchio A. 2010. Dissecting the role of MPS1 in chromosome biorientation and the spindle checkpoint through the small molecule inhibitor reversine. J Cell Biol 190:73-87. 10.1083/jcb.201001036

Shao X, Lai D, Zhang L, and Xu H. 2016. Induction of Autophagy and Apoptosis via PI3K/AKT/TOR Pathways by Azadirachtin A in Spodoptera litura Cells. Sci Rep 6:35482. 10.1038/srep35482

Peer) reviewing PDF | (2020:05:49288:2:0:NEW 30 Nov 2020) 
392

393

394

395

396

397

398

399

400

401

402

403

404

405

406

407

408

409

410

411

412

413

414

415

416

417

418

419

420

421

422

423

424

425

426

427

428

Sripa B, Kaewkes S, Sithithaworn P, Mairiang E, Laha T, Smout M, Pairojkul C, Bhudhisawasdi V, Tesana S, and Thinkamrop B. 2007. Liver fluke induces cholangiocarcinoma. PLoS medicine 4:e201.

Sripa B, Leungwattanawanit S, Nitta T, Wongkham C, Bhudhisawasdi V, Puapairoj A, Sripa C, and Miwa M. 2005. Establishment and characterization of an opisthorchiasis-associated cholangiocarcinoma cell line (KKU-100). World journal of gastroenterology: WJG 11:3392.

Sripa B, and Pairojkul C. 2008. Cholangiocarcinoma: lessons from Thailand. Curr Opin Gastroenterol 24:349-356. 10.1097/MOG.0b013e3282fbf9b3

Sripa B, Seubwai W, Vaeteewoottacharn K, Sawanyawisuth K, Silsirivanit A, Kaewkong W, Muisuk K, Dana P, Phoomak C, Lert-Itthiporn W, Luvira V, Pairojkul C, Teh BT, Wongkham S, Okada S, and Chamgramol Y. 2020. Functional and genetic characterization of three cell lines derived from a single tumor of an Opisthorchis viverrini-associated cholangiocarcinoma patient. Hum Cell. 10.1007/s13577-020-00334$\mathrm{W}$

Thorpe LM, Yuzugullu H, and Zhao JJ. 2015. PI3K in cancer: divergent roles of isoforms, modes of activation and therapeutic targeting. Nat Rev Cancer 15:7-24. 10.1038/nrc3860

Tsai YM, Wu KL, Chang YY, Hung JY, Chang WA, Chang CY, Jian SF, Tsai PH, Huang YC, Chong IW, and Hsu YL. 2020. Upregulation of Thr/Tyr kinase Increases the Cancer Progression by Neurotensin and Dihydropyrimidinase-Like 3 in Lung Cancer. Int J Mol Sci 21. 10.3390/ijms21051640

Tsuruta F, Masuyama N, and Gotoh Y. 2002. The phosphatidylinositol 3-kinase (PI3K)-Akt pathway suppresses Bax translocation to mitochondria. Journal of Biological Chemistry 277:14040-14047.

Watanapa P, and Watanapa W. 2002. Liver fluke-associated cholangiocarcinoma. British Journal of Surgery 89:962-970.

Wu Y-T, Tan H-L, Huang Q, Ong C-N, and Shen H-M. 2009. Activation of the PI3K-AktmTOR signaling pathway promotes necrotic cell death via suppression of autophagy. Autophagy 5:824-834.

Xu Q, Xu Y, Pan B, Wu L, Ren X, Zhou Y, Mao F, Lin Y, Guan J, Shen S, Zhang X, Wang C, Zhong Y, Zhou L, Liang Z, Zhao H, and Sun Q. 2016. TTK is a favorable prognostic biomarker for triple-negative breast cancer survival. Oncotarget 7:81815-81829. 10.18632/oncotarget.13245

Zhang W, Zhang JQ, Meng FM, and Xue FS. 2016. Dexmedetomidine protects against lung ischemia-reperfusion injury by the PI3K/Akt/HIF-1alpha signaling pathway. $J$ Anesth 30:826-833. 10.1007/s00540-016-2214-1

Peer] reviewing PDF | (2020:05:49288:2:0:NEW 30 Nov 2020) 
Figure 1

Reversine inhibited cell proliferation of KKU-100, KKU-213A and KKU-213B CCA cell lines and had a minor effect on an immortalized cholangiocyte cell line, MMNK1.

Cells were treated with increasing concentrations ( $\log _{10}$ scale) of reversine for $(A) 24,(B) 48$ and (C) $72 \mathrm{~h}$. Cells treated with $0.1 \%$ DMSO were used as a vehicle control. The cell viability was determined using the MTT assay. Data are mean \pm SD of three independent experiments. $* P<0.05$ are shown in all cell lines when compared to vehicle.

A

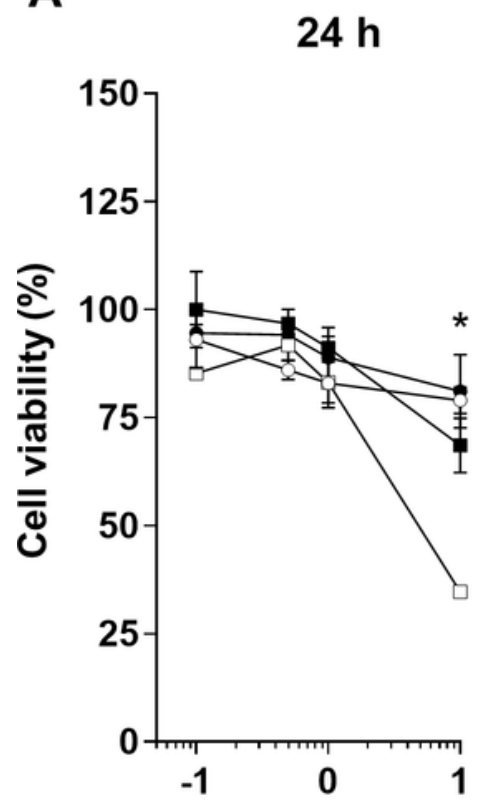

B

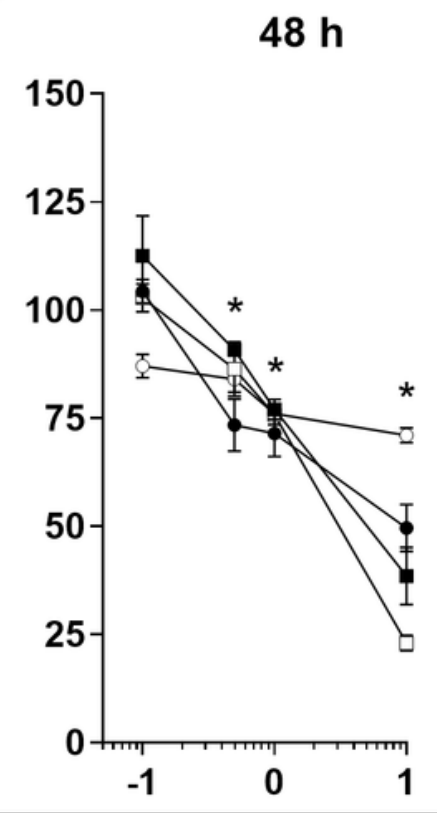

C

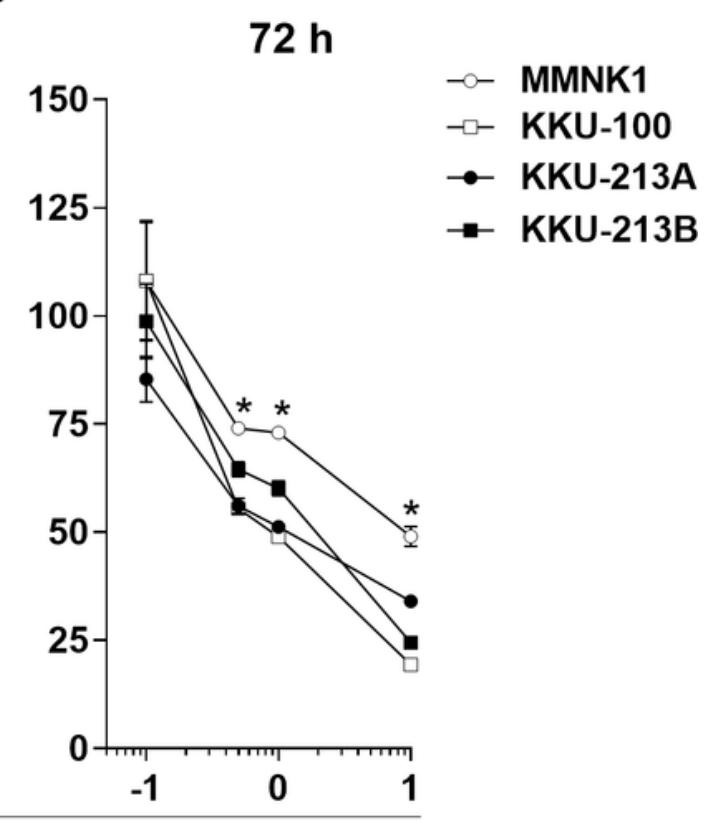

$\log _{10}[$ Reversine conc. $(\mu \mathrm{M})]$ 


\section{Figure 2}

Reversine induced G2/M cell cycle arrest and apoptosis. (A) Representative flow cytometry histograms of CCA cell lines treated with various concentrations of
reversine for $24 \mathrm{~h}$. The percentage of cells in each cell cycle phase are shown as mean \pm SD from two independent experiments (B) KKU-213A and (C) KKU-213B. (D) Western blot analyses of the expression of cell cycles regulating proteins and apoptosis-related proteins upon reversine treatment. Cells were treated with $4 \mu \mathrm{M}$ of reversine for $12,24,48$ and $72 \mathrm{~h}$. GAPDH was used as the internal control. The protein expression levels are reported as fold changes to vehicle control which was set as 1. (E) KKU-213A and (F) KKU-213B. Data shown are mean \pm SD of three separate experiments. $* P<0.05$ compared to vehicle control. 
A
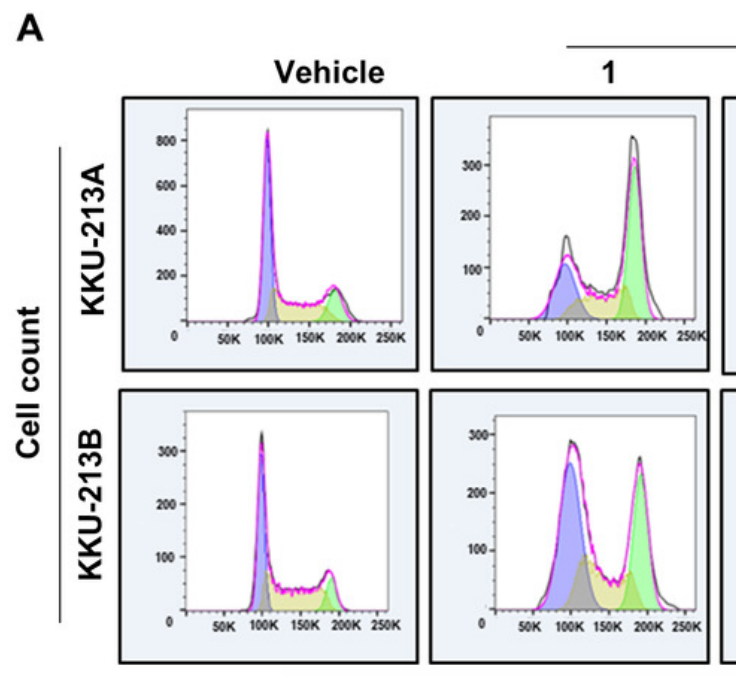

Reversine $(\mu \mathrm{M})$
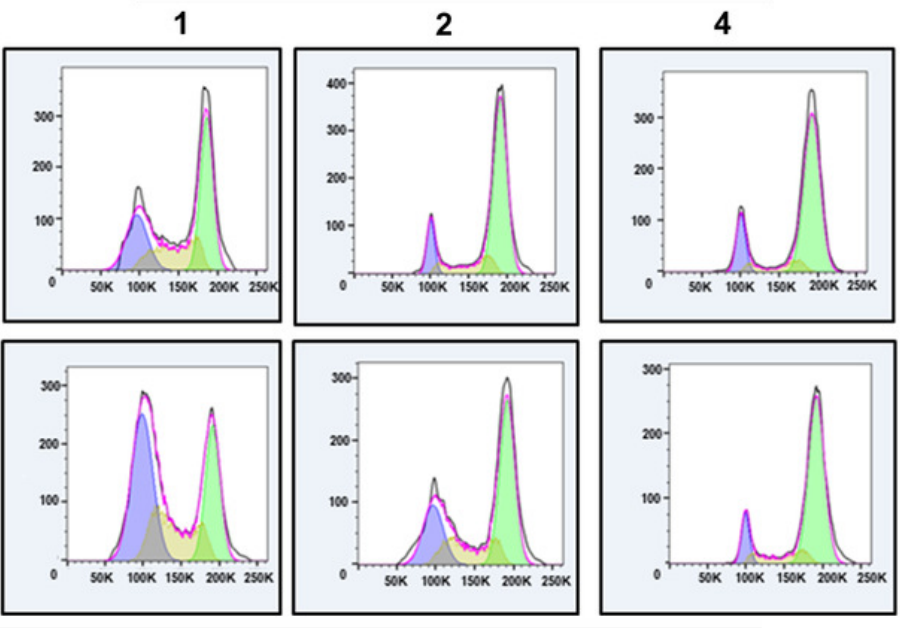

DNA content
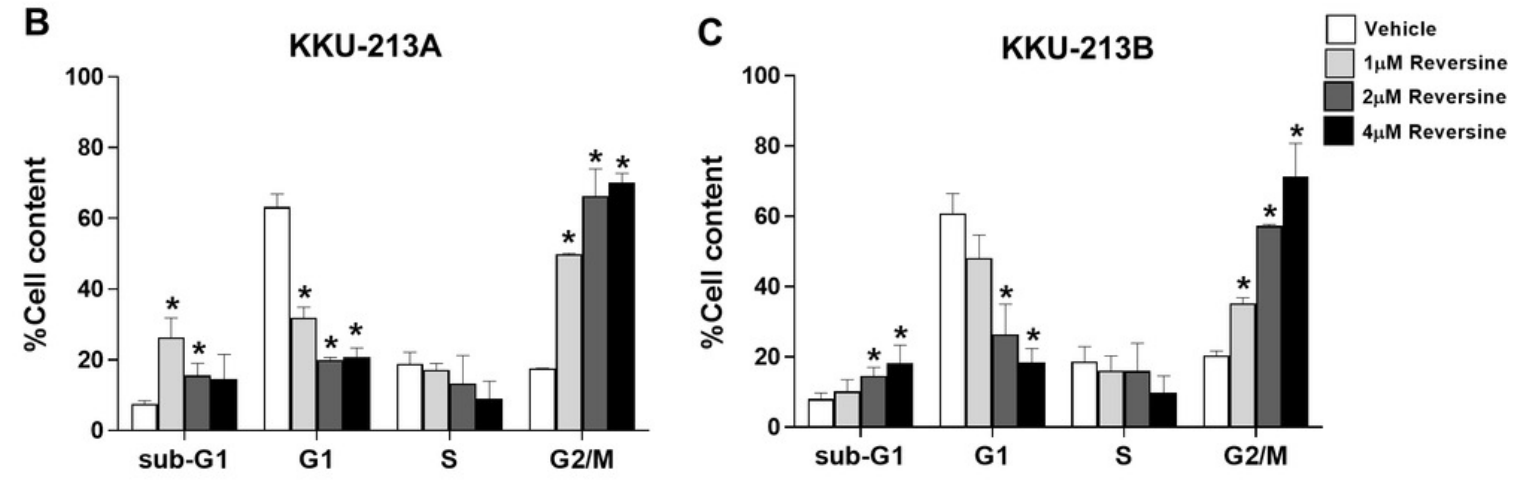

D KKU-213A

KKU-213B

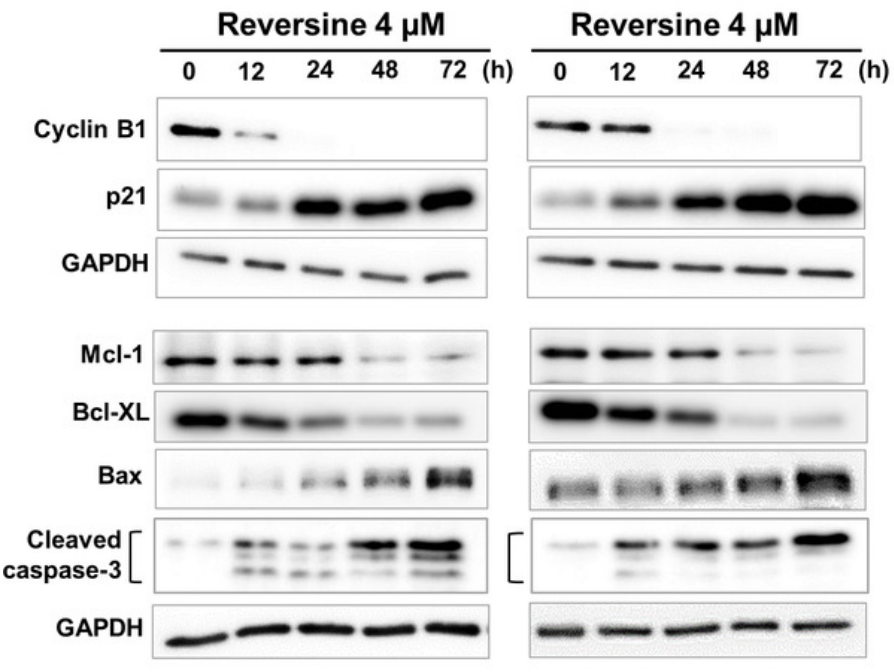

E

KKU-213A
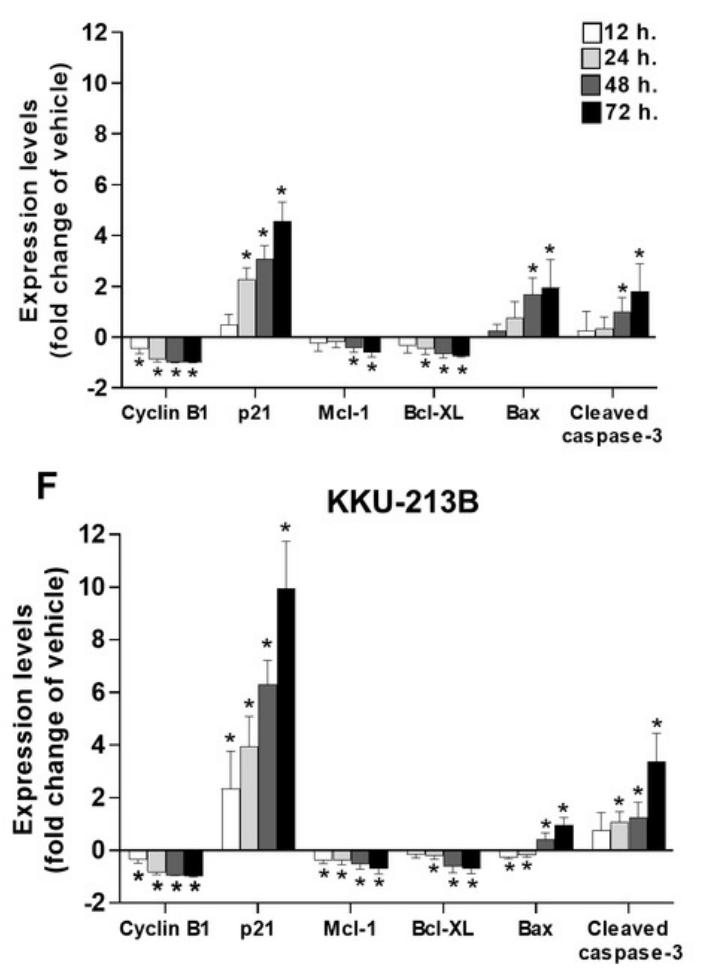


\section{Figure 3}

Reversine induced autophagy via reduction of glucose uptake and ATP production.

(A) Cellular morphology of KKU-213A and KKU-213B cells after reversine treatment for 12 and $24 \mathrm{~h}$ were observed under phase contrast microscopy with $200 \times$ magnification. The red arrows indicate the cytoplasmic vacuoles. (B) Western blot was performed to detect the expression of PI3K/AKT signalling proteins, HIF-1 $\alpha$, GLUT1, Beclin 1, LC3II/I and p62. GAPDH detected in the same experiment as Figure 2D was used as the internal control. The bar graphs represent the mean $\pm S D$ of expression levels (fold change to vehicle) from three independent experiments in (C) KKU-213A and (D) KKU-213B cell lines. (E) Glucose uptake and (F) ATP production were measured after reversine treatment at 12 and $24 \mathrm{~h}$. Data are represented as mean $\pm \mathrm{SD}$ of three independent experiments. $P$-values were calculated by Student's t-test compared with vehicle $(* P<0.05)$. 

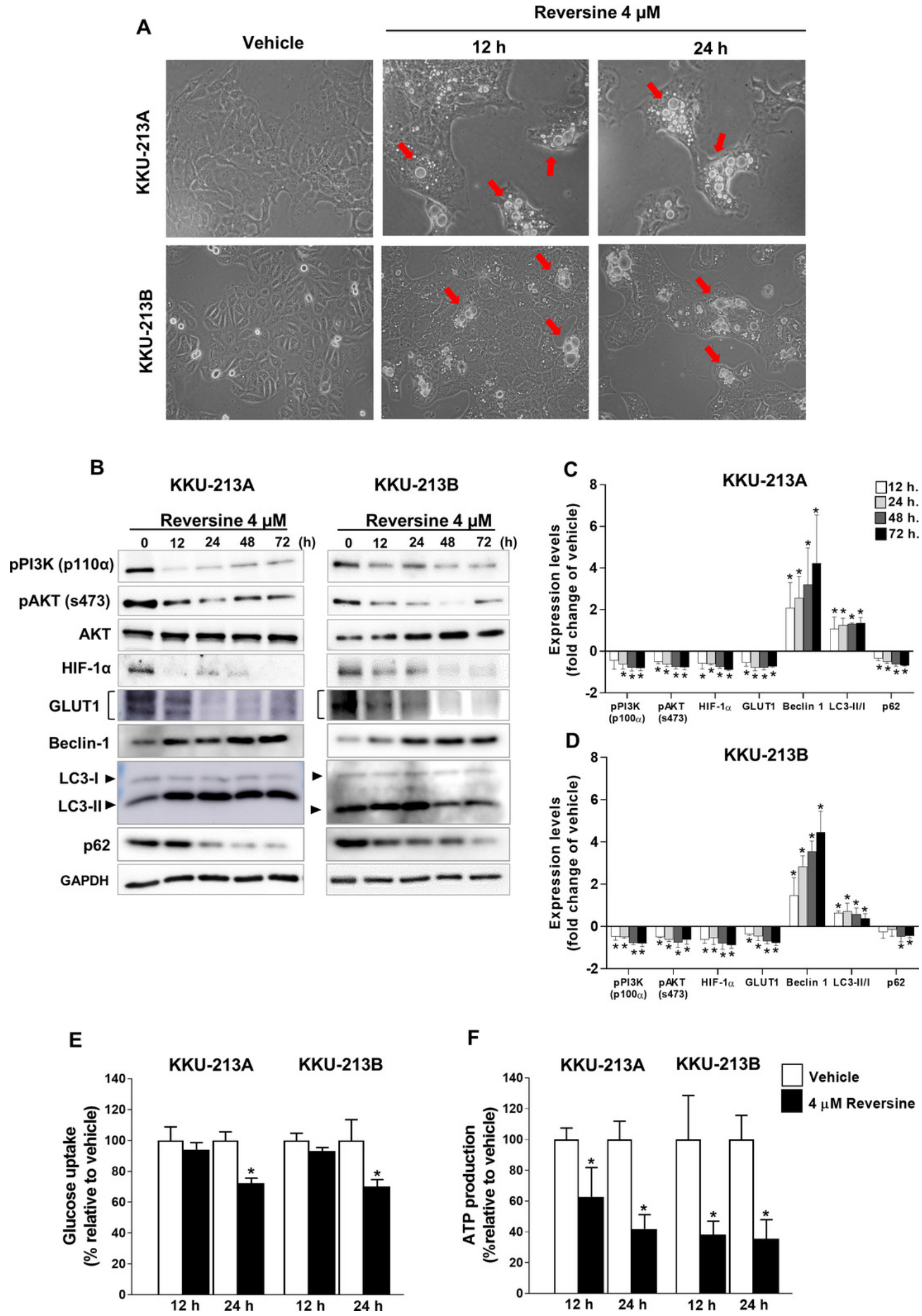

Peer) reviewing PDF | (2020:05:49288:2:0:NEW 30 Nov 2020) 


\section{Figure 4}

Reversine induced autophagy via suppression of AKT signaling.

Cellular morphology of (A) KKU-213A and (B) KKU-213B cells after reversine combined with SC-79 treatment for $24 \mathrm{~h}$ were observed under a phase contrast microscopy with $200 \mathrm{x}$ magnification. The red arrows indicate the cytoplasmic vacuoles. (C) Western blot was performed to detect the expression of pAKT/AKT, HIF-1 $\alpha$, GLUT1, Beclin 1, LC3II/I and p62. GAPDH was used as the internal control. The bar graphs represent the mean \pm SD of expression levels (fold change to vehicle) from three independent experiments in (D)

KKU-213A and (E) KKU-213B cells. ${ }^{*} P<0.05$ compared to vehicle and ${ }^{\#} \mathrm{P}<0.05$ compared to reversine alone. 
A

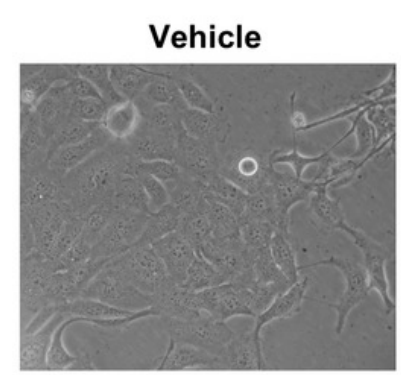

$4 \mu \mathrm{M} \mathrm{SC}-79$

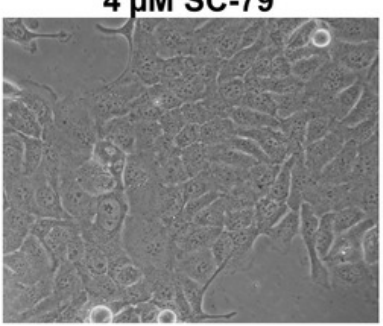

KKU-213A

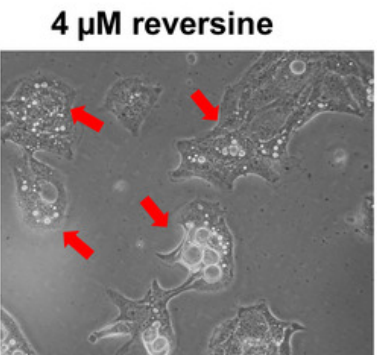

reversine + SC-79

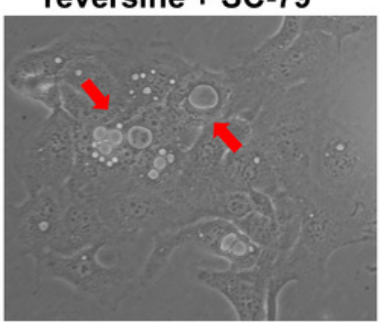

B

KKU-213B

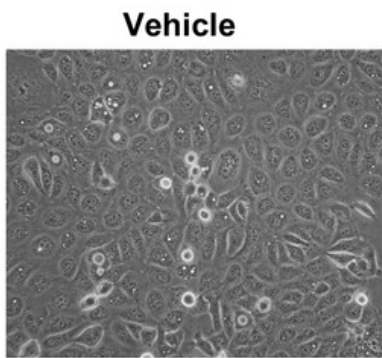

$4 \mu \mathrm{M} \mathrm{SC}-79$

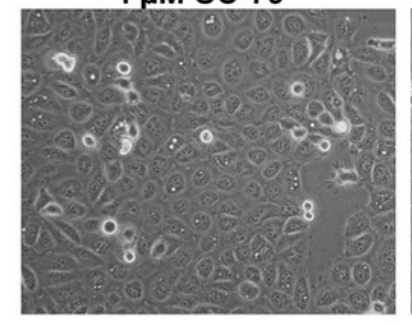

reversine + SC-79
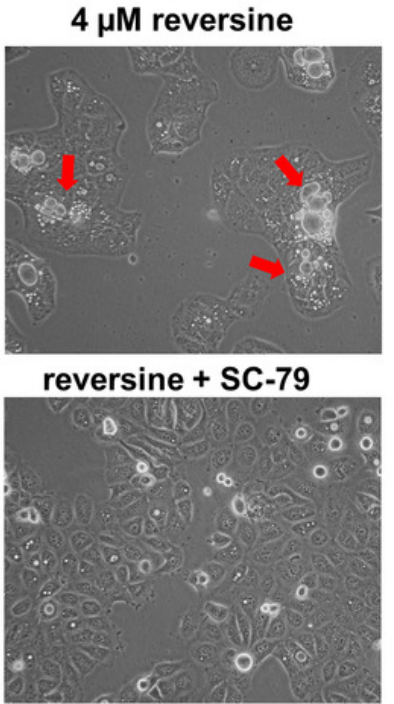

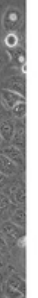

C

KKU-213A

KKU-213B
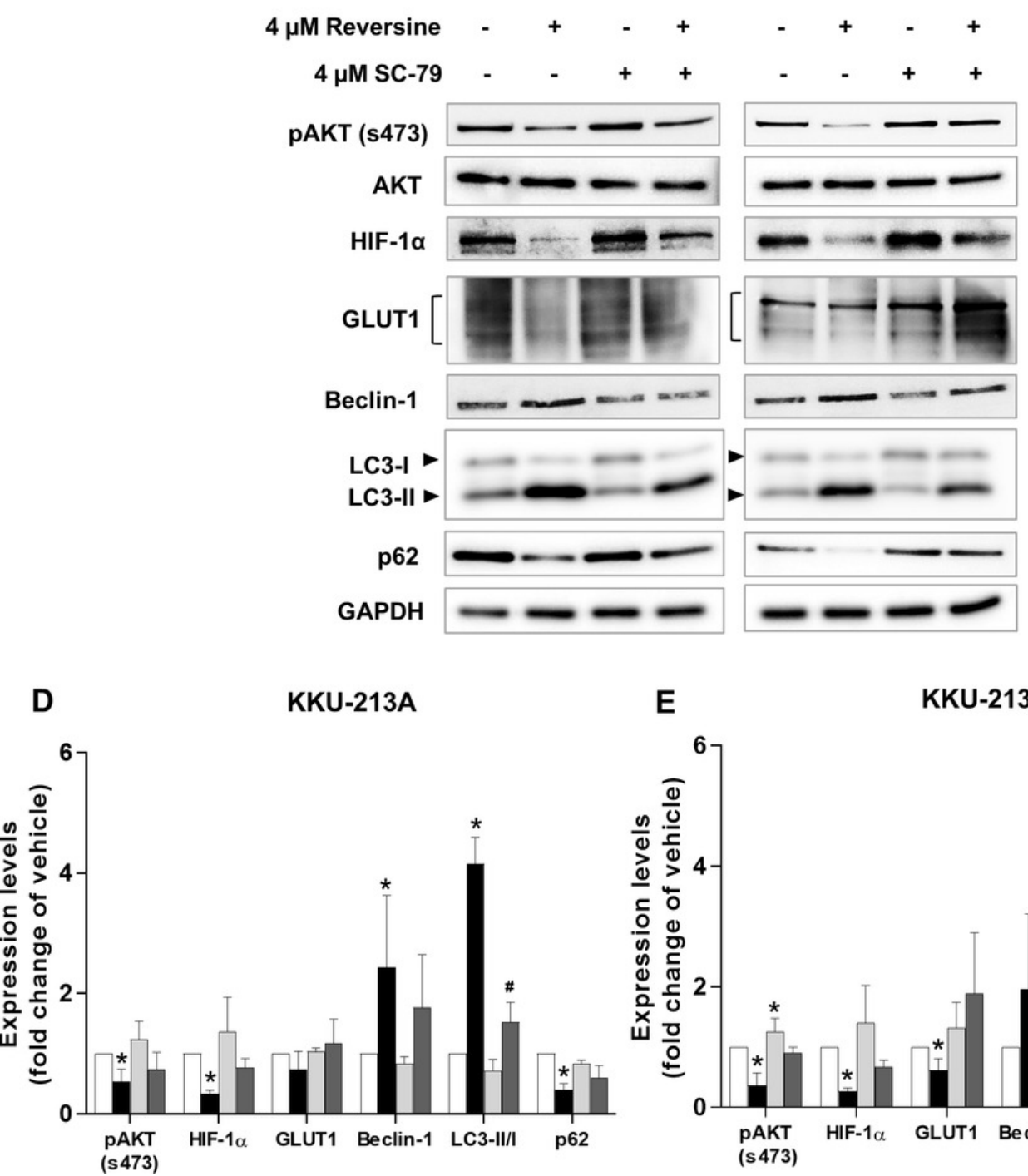

E

KKU-213B 
Figure 5

The proposed molecular mechanisms of anti-tumor effects of reversine on CCA cells.

Reversine, an MPS1 inhibitor, inhibited cell proliferation of CCA cells by multiple mechanisms including G2/M arrest with changes of cyclin B1 and p21. This inhibitor caused autophagy activation and autophagic flux via alterations of autophagy related proteins. Additionally, reversine-mediated autophagy partially inactivated the AKT signalling pathway. Reversine also reduced the glucose uptake and ATP production via suppression of HIF-1 $\alpha$ and GLUT1 expression. It consequently triggered apoptosis by decreasing of Bcl-XL and Mcl-1 expressions which subsequently inhibited Bax and activated the cleavage of caspase 3 .

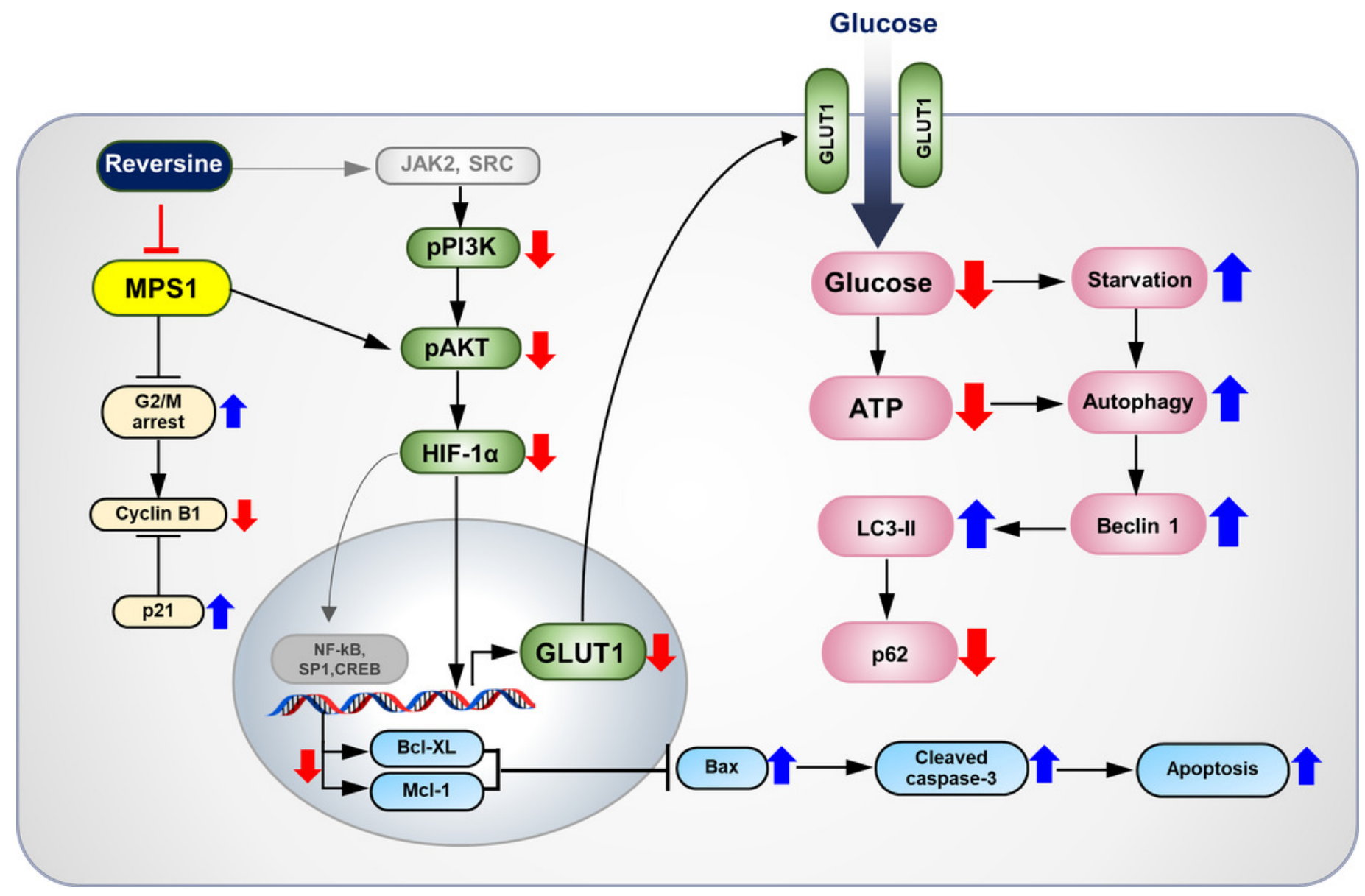




\section{Table $\mathbf{1}$ (on next page)}

$\mathrm{IC}_{50}$ of reversine in three CCA cell lines, KKU-100, KKU-213A and KKU-213B and an immortalized cholangiocyte cell line, MMNK1.

$I \mathrm{C}_{50}$ : Half-maximal inhibitory concentration. $* P$-value $<0.05$ compared with MMNK1 cell line at the same time points. 
2

\begin{tabular}{|c|c|c|c|c|}
\hline \multirow{2}{*}{ Time (h) } & \multicolumn{4}{|c|}{$\mathrm{IC}_{50}(\boldsymbol{\mu M})$} \\
\hline & MMNK1 & KKU-100 & KKU-213A & KKU-213B \\
\hline 24 & $>20$ & $5.51 \pm 0.38$ & $>10$ & $>10$ \\
\hline 48 & $16.71 \pm 1.81$ & $2.72 \pm 0.32 *$ & $6.83 \pm 2.61 *$ & $4.67 \pm 0.77 *$ \\
\hline 72 & $7.05 \pm 2.01$ & $0.62 \pm 0.42 *$ & $1.76 \pm 0.94 *$ & $1.75 \pm 0.82 *$ \\
\hline
\end{tabular}

